\title{
[Letter to the Editor] Isolation of mitochondria is necessary for precise quantification of mitochondrial DNA damage in human carcinoma samples
}

According to estimates made by the International Agency for Research on Cancer, the second leading cause of cancer deaths worldwide is liver cancer (1). Hepatocellular carcinoma (HCC) is the most common form of liver cancer, and it represents the third most common cause of cancer mortality worldwide, with an estimated half-million new cases diagnosed each year (2). The molecular mechanisms mediating carcinogenesis in HCC, which have not been fully elucidated, represent the final stage of a longstanding liver disease that usually begins with an acute hepatic insult that progresses to fibrosis and cirrhosis, ultimately leading to hepatocyte transformation (3). Several laboratories are actively working to characterize the mechanisms responsible for hepatocyte transformation and the role of mitochondria in liver tumorigenesis. In the past decade, somatic mitochondrial DNA (mtDNA) mutations have been identified in several types of cancer (4), including HCC $(5,6)$, and these mutations are believed to cause mitochondrial dysfunction, increase the production of reactive oxygen species (ROS), and promote tumor growth, contributing to the onset of a vicious cycle $(7,8)$. This accounts for the renewed interest in mitochondrial biology and in methods for studying mtDNA damage and mechanisms of mtDNA repair.

The most widely used method to measure nuclear DNA (nDNA) and mtDNA damage in mammalian cells is quantitative-PCR (Q-PCR) (9). The Q-PCR assay for DNA damage is based on the principle that many kinds of DNA lesions can slow down or block the progression of DNA polymerase during PCR amplification (10). Therefore, if equal amounts of DNA from differently treated samples are Q-PCR-amplified under identical conditions, DNA with fewer lesions will amplify to a greater extent than more damaged DNA. In the protocol proposed by Furda et al. (9), total DNA isolated from cells or tissue is used as the template to measure mtDNA damage. As part of their protocol, the amplification of a short mitochondrial fragment ( 100 bp), where the probability of base damage is very low, is used to calculate the relative amount of mtDNA copies between different samples and to normalize the lesion frequencies calculated from a long $(\sim 10$ kb) mitochondrial fragment. However, high levels of mtDNA damage could be underestimated using this approach because the short fragment may also contain damaged bases that slow down or block the action of the polymerase, thereby altering the quantification of the mtDNA template.

To normalize the mtDNA copy number between different samples, Ballinger et. al used the ratio of nDNA to mtDNA (11). However, this approach could also lead to inaccurate results. For example, it has been demonstrated that tumor cells are subjected to chromosomal rearrangements that in the case of HCC could lead to differences of $85 \%$ in terms of nDNA content with respect to healthy tissues (12). Here, we demonstrate that to accurately measure the level of mtDNA damage in HCC tumor tissue, it is necessary to isolate mitochondria and use mtDNA as the Q-PCR template, thereby avoiding the need to amplify a short mtDNA fragment to normalize the amount of mtDNA.

Human biopsies derived from a tumorous liver mass (HCC) and from a non-tumorous (distal) section from the same patient were processed as previously described (13). Liver tissue was immersed in $50 \mathrm{~mL}$ ice-cold IBC buffer, rinsed 2 times to completely remove blood, cut into small pieces, and transferred to a Potter-Elvehjem tissue grinder. Next, $7 \mathrm{~mL}$ IBc buffer was added, and the liver was homogenized using a Teflon pestle at the minimum speed until the suspension was homogeneous. This process leads to the disruption of liver tissue and the lysis of cells. The homogenate was then transferred to a $15 \mathrm{~mL}$ plastic tube and centrifuged at $70 \times g$ for 3 min at $4^{\circ} \mathrm{C}$ to separate non-homogenized tissue pieces. The supernatant was transferred to a $15 \mathrm{~mL}$ Falcon tube and centrifuged at $600 \times g$ for $10 \mathrm{~min}$ at $4^{\circ} \mathrm{C}$ to pellet intact nuclei that were then treated separately. The supernatant, containing mitochondria, was transferred to a $15 \mathrm{~mL}$ glass centrifuge tube and centrifuged at $7000 \times g$ for $10 \mathrm{~min}$ at $4^{\circ} \mathrm{C}$. The supernatant was discarded, and the pellet, containing mitochondria, was washed with $5 \mathrm{~mL}$ IBC buffer, centrifuged at $7000 \times g$ for 10 min at $4^{\circ} \mathrm{C}$, and resuspended in 800 $\mu \mathrm{L} \mathrm{IBC}$ buffer; this was the mitochondrial fraction (MF). The mitochondria were finally washed with IBc buffer containing $1 \mathrm{M} \mathrm{KCl}$ to eliminate cytoplasmic proteins and contaminant nDNA (14).

The pellet of nuclei was washed by gentle resuspension in $\mathrm{T} 1$ solution and centrifuged at $600 \times g$ for $10 \mathrm{~min}$ at $4^{\circ} \mathrm{C}$. To obtain nuclear protein extract, the pellet was lysed in T2 solution, incubated on ice for $30 \mathrm{~min}$, and then centrifuged at $14,000 \times g$ for $20 \mathrm{~min}$ to remove cell membranes. The supernatant was collected as the nuclear protein fraction (NF). For isolation of nDNA, after washing with T1 solution, the pellet was lysed with TRI Reagent, and $\mathrm{nDNA}$ was isolated as reported in the Supplementary Material (Figure 1A). 
A Homogenization of the tissue in IBC solution and

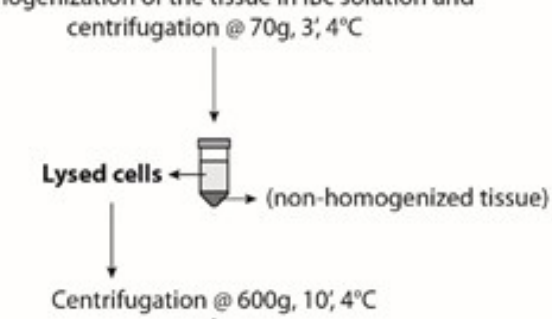

Figure 1. Human liver subcellular fractionation. (A) Workflow for subcellular fractionation of liver tissue. (B) Western blot analysis of mitochondrial (MF) and nuclear (NF) fractions from human liver tissues. Samples were separated by $12 \%$ SDSPAGE and analyzed by western blotting to evaluate the purity of the indicated fractions. ATP synthase complex $V$ (ATP5A) and lysine (K)-specific demethylase 1A (LSD1) were used as mitochondrial and nuclear markers, respectively. (C) Agarose gel electrophoresis analysis of human mtDNA extracted from mitochondria from hepatocellular carcinoma (HCC) biopsies.
B

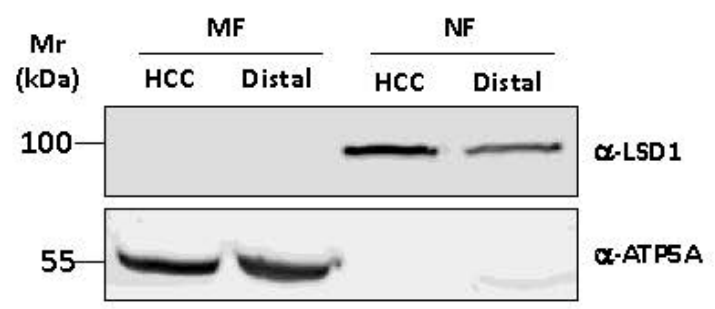

Wash in $\mathrm{T} 1$ solution and centrifugation (\$) $600 \mathrm{~g}, 10,4^{\circ} \mathrm{C}$

Centrifugation@7000g, $10^{\circ}, 4^{\circ} \mathrm{C}$

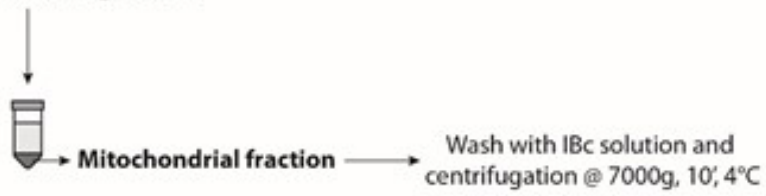
centrifugation @ $7000 \mathrm{~g}, 10^{\circ}, 4^{\circ} \mathrm{C}$

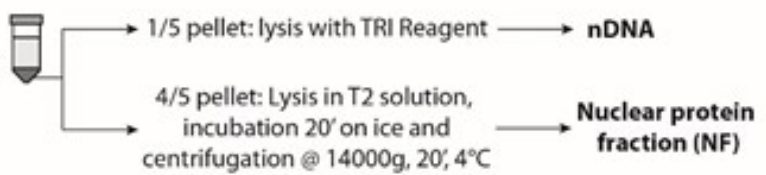

$2 \mathrm{mg}$ mitochondria: $\mathrm{IBC}+1 \mathrm{M} \mathrm{KCl}$

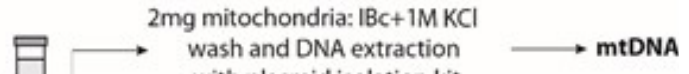
with plasmid isolation kit

Mitochondrial fraction (MF)

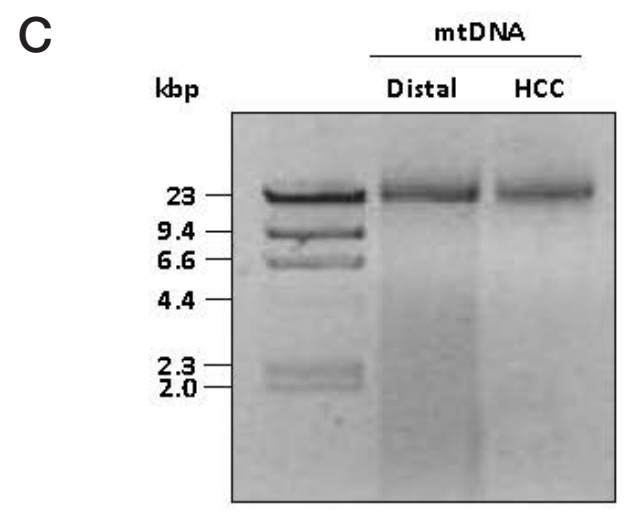

For western blot analysis of mitochondrial (MF) and nuclear (NF) fractions from human HCC biopsies, mitochondrial membrane ATP synthase complex $V$ (ATP5A) and lysine (K)-specific demethylase 1A (LSD1) were used as the mitochondrial and nuclear markers, respectively. Western blot analysis confirmed the possibility of isolating the two subcellular compartments without apparent crosscontamination (Figure 1B).

Genomic DNA from HCC samples was isolated from nuclei ( 200 mg of nDNA per $1 \mathrm{~g}$ of liver tissue). To purify the circular, $16-\mathrm{kb}$ mtDNA, we used a plasmid isolation kit (15). Two milligrams of isolated mitochondria were used to extract mtDNA from HCC and non-tumor tissues ( 450 ng of mtDNA per $1 \mathrm{~g}$ of liver tissue) and quantified with PicoGreen reagent (Supplementary Figure S1). Figure 1C shows an image of an agarose gel in which $150 \mathrm{ng}$ of mtDNA from the distal area and the HCC tissue of Patient 1 were separated, showing the presence of a single band of $\sim 16 \mathrm{~kb}$. To confirm the integrity of the isolated nDNA and the absence of nDNA contamination in the mtDNA preparation, we amplified a region of the human GAPDH gene by RT-PCR. We used 100 ng of nDNA as the positive control; $10 \mathrm{pg}$ of mtDNA, which corresponds to 10 times the number of base pairs of nDNA (considering the nDNA as a unique molecule); and a reaction without template as the negative control. The nDNA amplified with $C_{q}$ values of 16.79 and 16.93 for the distal and tumor tissues, respectively, while the $C_{q}$ values of mtDNA from the distal and tumor regions were 31.15 and 29.70, respectively. The amplification of the negative control without template was not detectable. We demonstrated that through this approach it is possible to purify nuclei and mitochondria and also isolate highquality mtDNA without contamination by $n D N A$.

In contrast to commonly used protocols for the evaluation of DNA damage, where the mtDNA is not separated from the genomic DNA (9), we used mtDNA from tumor and distal liver regions isolated from four patients to evaluate the levels of DNA damage in HCC mitochondria with respect to healthy tissue. In Figure 2A, the gel image and the quantification of relative band intensity for the $\mathrm{Q}-\mathrm{PCR}$ analysis of the sample from Patient 1 
A

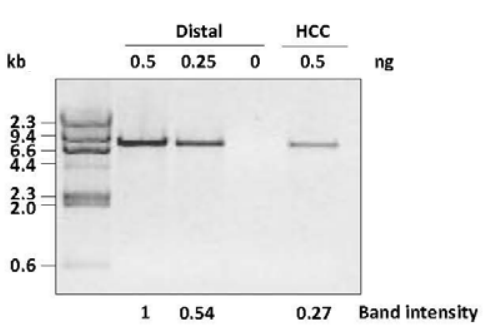

C

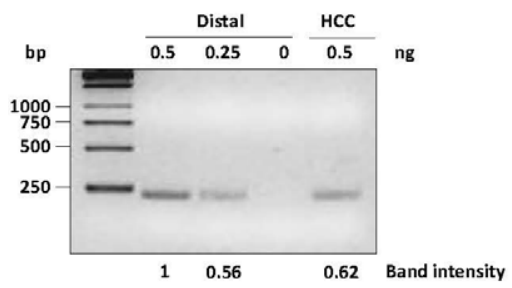

D

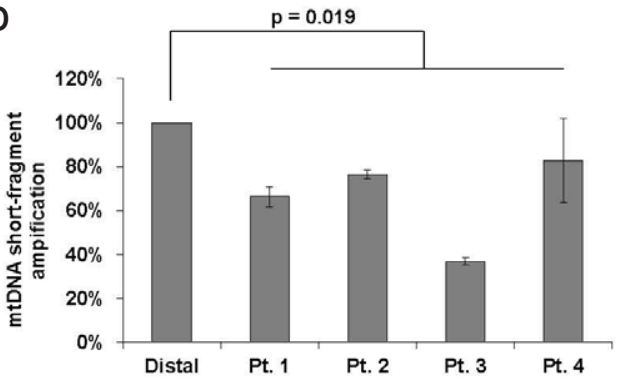

E

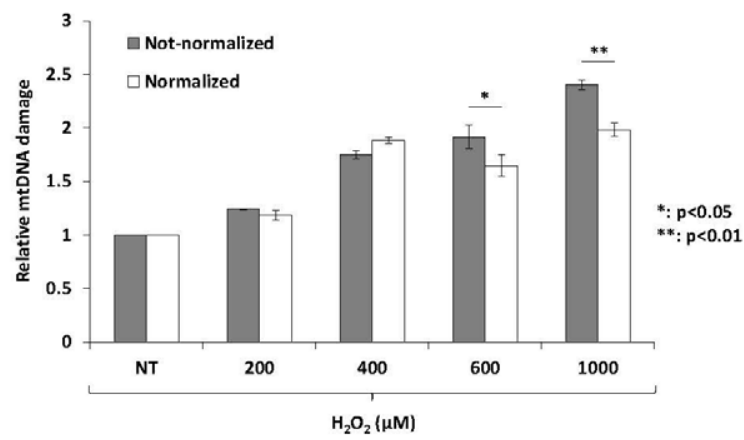

$\mathbf{F}$

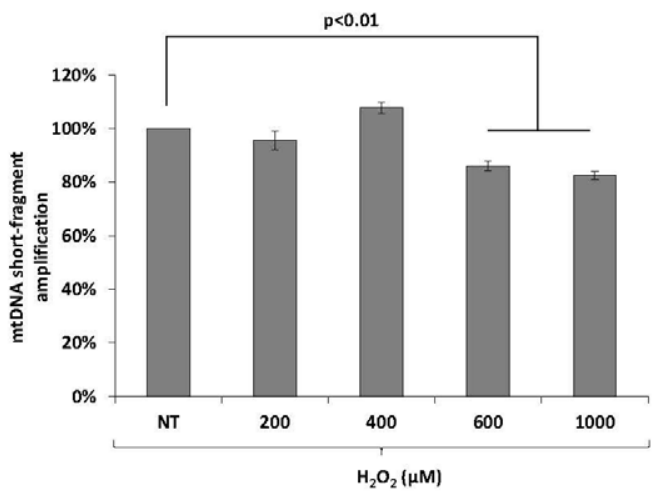

Figure 2. Electrophoresis and quantification of Q-PCR products for comparative mtDNA damage analysis. (A) Representative gel image of the Q-PCR analysis of the mtDNA long fragment. mtDNA from hepatocellular carcinoma (HCC) tumor and nontumor distal tissues of Patient 1 (Pt. 1) were used as template. Amplicons were separated on a $0.8 \%$ agarose gel, and the band intensity of each lane was measured. (B) Histogram showing the relative mtDNA damage analysis of HCC tumor tissues for all four patients. Q-PCR was performed, and levels of mtDNA damage in HCC samples were calculated in respect to non-tumor tissue. (C) Representative gel image of the Q-PCR analysis of the short mtDNA fragment. mtDNA from HCC tumor and non-tumor distal tissues of Pt. 1 was used as template. Fragments were separated on a $2 \%$ agarose gel, and the band intensity of each lane was measured. (D) Histogram of the efficiency of mtDNA short fragment amplification all four patients. (E) Comparison of mtDNA damage analysis of HeLa cells treated with $\mathrm{H}_{2} \mathrm{O}_{2}$, with the amount of the long mitochondrial Q-PCR amplicons normalized (white bars) or not normalized (gray bars) to the short mtDNA amplicons. mtDNA damage was calculated as the inverse of mtDNA long fragment amplification. (F) Efficiency of HeLa cell mtDNA short fragment amplification after $\mathrm{H}_{2} \mathrm{O}_{2}$ treatment. are shown as an example. To verify the quantitative amplification conditions, 1 reaction containing 50\% (0.25 ng) of the mtDNA template $(0.5 \mathrm{ng})$ and 1 reaction without sample $(0 \mathrm{ng})$ were compared. To this end, fluorescence readings of the $\mathrm{Q}$-PCR reactions were quantified in triplicate with PicoGreen reagent and then averaged for each sample. These raw readings were then background-corrected by subtracting the blank values (9). The relative amplification of the mIDNA was determined by calculating the ratio of the fluorescence readings obtained for the tumor tissue to those of the distal section for each patient sample. Relative mtDNA damage was then expressed as the inverse of this relative amplification of the mtDNA long fragment. In all cases, the mtDNA purified from mitochondria of the tumor tissue amplified with lower efficiency, an indication of higher levels of damage compared with the mtDNA derived from the distal section ( $P=$ 0.019) (Figure 2B).

We next amplified a short fragment of $221 \mathrm{bp}$ that is typically used to normalize the amount of mtDNA when nDNA and mtDNA are not separated during extraction. To verify quantitative amplification conditions, we examined 1 reaction containing $50 \%(0.25 \mathrm{ng})$ of the mtDNA template $(0.5 \mathrm{ng})$ and 1 reaction containing no sample (0 $\mathrm{ng})$. In Figure 2C, the short amplicons of the distal and HCC mtDNA of Patient 1 are visible, and the band analysis shows an unexpected reduction of $38 \%$ for the $\mathrm{HCC}$ amplicon with respect to the distal section mtDNA. Next, the Q-PCR reactions for the short mtDNA fragment from all 4 patients were quantified with PicoGreen reagent, confirming the presence of high levels of base damage in the HCC samples $(P=0.019)$ (Figure 2D).

To test the hypothesis that high levels of mtDNA damage could affect amplification of the short amplicon, thereby altering quantification of mtDNA damage levels, we treated HeLa cells with increasing amounts of $\mathrm{H}_{2} \mathrm{O}_{2}$ for 15 min and then measured the levels of mtDNA damage. The histogram in Figure 2E shows quantification of the long mitochondria Q-PCR amplicons normalized or not normalized to the short PCR fragment. 
Normalized measurements (white bars) show an initial increase in mtDNA damage, which reaches its maximum after treatment with $400 \mu \mathrm{M}$ of $\mathrm{H}_{2} \mathrm{O}_{2}$ and unexpectedly does not increase with higher concentrations of $\mathrm{H}_{2} \mathrm{O}_{2}$. In contrast, by not normalizing to the short mtDNA amplicon (gray bars), we observed a significant concentrationdependent increase in DNA damage (Figure 2E). The reduced amplification efficiency of the short mitochondrial $\mathrm{PCR}$ fragment in cells treated with $600 \mu \mathrm{M}$ or $1 \mathrm{mM} \mathrm{H}_{2} \mathrm{O}_{2}$ (Figure 2F) reveals a bias in measurement of mtDNA base lesions when the sample is normalized for the short amplicon. These data confirm the hypothesis that in samples with a high level of damaged bases, such as tumor tissues, a short mtDNA amplicon should not be used to normalize the amount of mtDNA for the calculation of mtDNA damage.

In 2014, the Van Houten group published an updated Q-PCR-based method for measuring nDNA and mtDNA damage in mammalian cells that does not require the isolation of mtDNA separately from genomic DNA (9). This undoubtedly represents the main strength of the assay because the labor-intensive step of mitochondrial isolation is not needed, and it also provides information about mtDNA copy number. However, our data demonstrate that high levels of mtDNA damage may be underestimated because damage to DNA bases could occur also in the short amplicon, thereby altering the quantification of the relative mtDNA copy number (Figure 2, D-F). The present study supports the hypothesis that in samples with a high level of damaged bases, such as tumor tissues, it is not possible to use total DNA for calculation of mtDNA damage.

\section{Author contributions}

C.V. and A.B. defined the research theme, designed methods, carried out the laboratory experiments, analyzed the data, interpreted the results and wrote the paper. U.B. provided the human biopsies. B.B. and G.T. contributed to data interpretation and discussion of the results.
Acknowledgments

This work was funded by grants from Associazione Italiana per la Ricerca sul Cancro AIRC (MFAG 2015 Id.16780) to C.V.; and the U.S.-Italy Fulbright Commission and the University of Udine to B.B.

\section{Competing interests}

The authors declare no competing interests.

\section{References}

1. Torre, L.A., F. Bray, R.L. Siegel, J. Ferlay, J. Lortet-Tieulent, and A. Jemal. 2015. Global cancer statistics, 2012. CA Cancer J. Clin. 65:87-108.

2. Govaere, O. and T. Roskams. 2015. Pathogenesis and prognosis of hepatocellular carcinoma at the cellular and molecular levels. Clin. Liver Dis. 19:261-276.

3. Zhu, A.X. 2003. Hepatocellular carcinoma: are we making progress? Cancer Invest. 21:418428.

4. Lee, H.C., C.M. Chang, and C.W. Chi. 2010. Somatic mutations of mitochondrial DNA in aging and cancer progression. Ageing Res. Rev. 9(Supp/ 1):S47-S58.

5. Yin, P.H., H.C. Lee, G.Y. Chau, Y.T. Wu, S.H. Li, W.Y. Lui, Y.H. Wei, T.Y. Liu, and C.W. Chi. 2004. Alteration of the copy number and deletion of mitochondrial DNA in human hepatocellular carcinoma. Br. J. Cancer 90:2390-2396.

6. Yin, P.H., C.C. Wu, J.C. Lin, C.W. Chi, Y.H. Wei, and H.C. Lee. 2010. Somatic mutations of mitochondrial genome in hepatocellular carcinoma. Mitochondrion 10:174-182.

7. Penta, J.S., F.M. Johnson, J.T. Wachsman, and W.C. Copeland. 2001. Mitochondrial DNA in human malignancy. Mutat. Res. 488:119133.

8. Wallace, D.C. 2012. Mitochondria and cancer. Nat. Rev. Cancer 12:685-698.

9. Furda, A., J.H. Santos, J.N. Meyer, and B. Van Houten. 2014. Quantitative PCR-based measurement of nuclear and mitochondrial DNA damage and repair in mammalian cells. Methods Mol. Biol. 1105:419-437.

10. Ponti, M., S.M. Forrow, R.L. Souhami, M. D'Incalci, and J.A. Hartley. 1991. Measurement of the sequence specificity of covalent DNA modification by antineoplastic agents using Taq DNA polymerase. Nucleic Acids Res. 19:2929-2933.

11. Ballinger, S.W., T.G. Bouder, G.S. Davis, S.A. Judice, J.A. Nicklas, and R.J. Albertini. 1996. Mitochondrial genome damage associated with cigarette smoking. Cancer Res. 56:5692-5697.

12. Zimmermann, U., D. Feneux, G. Mathey, F. Gayral, D. Franco, and P. Bedossa. 1997. Chromosomal aberrations in hepatocellular carcinomas: relationship with pathological features. Hepatology 26:1492-1498.

13. Frezza, C., S. Cipolat, and L. Scorrano. 2007. Organelle isolation: functional mitochondria from mouse liver, muscle and cultured fibroblasts. Nat. Protoc. 2:287-295.

14. Lee, K.W. and D.F. Bogenhagen. 2016. Scalable Isolation of Mammalian Mitochondria for Nucleic Acid and Nucleoid Analysis. Methods Mol. Biol. 1351:67-79.

15. Quispe-Tintaya, W., R.R. White, V.N. Popov, J. Vijg, and A.Y. Maslov. 2013. Fast mitochondrial DNA isolation from mammalian cells for next-generation sequencing. Biotechniques 55:133-136.

Arianna Barchiesi', Umberto Baccarani', Blase Billack², Gianluca Tell', and Carlo Vascotto ${ }^{1}$

${ }^{1}$ Department of Medical and Biological Sciences, University of Udine, Udine, Italy and ${ }^{2}$ Department of Pharmaceutical Sciences, College of Pharmacy and Health Sciences, St. John's University, Jamaica, NY

BioTechniques 62:13-17 (January 2017)

doi 10.2144/000114491

Keywords: subcellular fractionation from liver tissue; mitochondrial DNA damage quantification; human hepatocellular carcinoma

Supplementary material for this article is available at www.BioTechniques.com/article/114491.

Received 02 May 2016; accepted 15 November 2016.

Address correspondence to Carlo Vascotto, Department of Medical and Biological Sciences, University of Udine, Udine, 33100, Italy. E-mail: carlo.vascotto@uniud.it

To purchase reprints of this article, contact: biotechniques@fosterprinting.com 